\title{
Shockwave consolidation of nanostructured thermoelectric materials
}

\author{
Narasimha S. Prasad ${ }^{* 1}$, Patrick Taylor ${ }^{2}$, and David Nemir ${ }^{3}$ \\ ${ }^{1}$ NASA Langley Research Center, 5 N. Dryden St., MS 468, Hampton VA, 23681 \\ ${ }^{2}$ US Army Research Laboratory, 2800 Powder Mill Road, Adelphi, MD 20783 \\ ${ }^{3}$ TXL Group, Inc. 2000 Wyoming Ave., El Paso, TX 79903
}

\begin{abstract}
Nanotechnology based thermoelectric materials are considered attractive for developing highly efficient thermoelectric devices. Nano-structured thermoelectric materials are predicted to offer higher ZT over bulk materials by reducing thermal conductivity and increasing electrical conductivity. Consolidation of nano-structured powders into dense materials without losing nanostructure is essential towards practical device development. Using the gas atomization process, amorphous nano-structured powders were produced. Shockwave consolidation is accomplished by surrounding the nanopowder-containing tube with explosives and then detonating. The resulting shock wave causes rapid fusing of the powders without the melt and subsequent grain growth. We have been successful in generating consolidated nanostructured bismuth telluride alloy powders by using the shockwave technique. Using these consolidated materials, several types of thermoelectric power generating devices have been developed. Shockwave consolidation is anticipated to generate large quantities of nanostructred materials expeditiously and cost effectively. In this paper, the technique of shockwave consolidation will be presented followed by Seebeck Coefficient and thermal conductivity measurements of consolidated materials. Preliminary results indicate a substantial increase in electrical conductivity due to shockwave consolidation technique.
\end{abstract}

Keywords: Thermoelectric effect, nano-structured materials, Shock wave consolidation, Bismuth telluride alloy.

\section{INTRODUCTION}

Space exploration missions have a critical need for compact, efficient, reliable, rugged and long-life electrical power generators. Radio Isotope Thermoelectric Generators (RTGs) have been time tested and successfully used by NASA since the NIMBUS days in 1969 to the CASSINI Mission in 1997. RTGs are the power sources of choice for missions involving extreme temperatures, temperature cycling, harsh radiation environment, lack of solar radiation and stringent weight, area and volume requirements. They are typically used when power requirements are less than 500 Watts. The earlier NASA missions have used RTGs with PbTe and SiGe as thermoelectric materials that had efficiencies in the range of 3-7\%.

Thermoelectric Power Generator (TPG) devices directly convert heat energy to electricity without moving parts ${ }^{1}$. NASA LaRC is working on several concepts to develop practical thermoelectric devices to harness waste heat using advancing nanotechnology based techniques combined with novel material synthesis and effective thermal management architectures. The goal is to increase the conversion efficiency by $2.5 \mathrm{x}$ or more. Prototype TE modules that utilize low temperature differentials have been developed.

Unlike the Peltier Effect that deals with heat liberation or absorption when current passes through two dissimilar conductors (depending upon polarity), the Seebeck Effect (Thermoelectric effect) is the generation of electrical current when two dissimilar conductor junction is subjected to a temperature gradient. Power Generators based on the Thermoelectric Effect, (a) harnesses waste heat, (b) are solid-state and hence do not involve moving parts, (c) a compact architecture with extended operational life, (d) the long life times (>20 years) with high reliability (those utilized in Cassini Mission with RTGs appear to be still working) and (e) are eco-friendly.

\footnotetext{
*narasimha.s.prasad@nasa.gov; Phone 757-864-9403; Fax 757-864-8828.
} 


\section{THERMOELECTRIC EFFECT}

Thermoelectric materials, in general, are classified into three temperature ranges of operation and they are: (a) Room Temp- 500K; materials include Bismuth Telluride and its alloys, (b) intermediate range spanning over 600-900K; materials include PbTe-based alloys and TAGS (Te-Ag-Ge-Sb), and (c) high temperature range 1000-1300K; materials include Si-Ge alloys. The main factors for the fabrication of high efficiency TPG devices are: (a) selection of high ZT materials, (b) providing high temperature electrical contacts, (c) incorporating efficient thermal management architecture for minimizing parasitic losses and (d) preventing material degradation at operating temperatures. The primary focus of research around the world, in general, is to engineer TE materials with high ZT, preferably greater than 1 and subsequently work on other factors as indicated above that are critical for fabrication of practical devices.

TPG efficiency is determined by the figure of merit ZT and it depends only on material properties.

$$
Z T=\frac{S^{2} \sigma}{k}(T)
$$

In Eq. $1, S$ is the Seebeck coefficient (thermopower $(\mu \mathrm{V} / \mathrm{K})$ ) that indicates how much average thermal energy is transported by each carrier, $\sigma$ is the electrical conductivity $(\mathrm{S} / \mathrm{m})$ and it indicates how much the carriers can transport energy without Joule loss, $\mathrm{k}$ is the thermal conductivity that defines how small the reverse flow of heat from the coldside to the hot-side opposing the electron-transport of heat, and T is the operating temperature. The term $\left(\mathrm{S}^{2} \sigma\right)$ is sometimes referred to as the power factor. Often, Eq. 1 is written as

$$
Z=\frac{\sigma S^{2}}{\kappa}=\frac{\sigma S^{2}}{\kappa_{e}+\kappa_{l}}
$$

Where $\kappa=$ Total thermal conductivity $(\mathrm{W} / \mathrm{mK}), \kappa_{\mathrm{e}}=$ electronic thermal conductivity $(\mathrm{W} / \mathrm{mK})$, and $\kappa_{1}=$ lattice thermal conductivity $(\mathrm{W} / \mathrm{mK})$.

When taking measurements of a thermoelectric material, it is not possible to measure $\kappa_{\mathrm{e}}$ and $\kappa_{\mathrm{ph}}$ separately. Instead, the total thermal conductivity, $\kappa=\kappa_{\mathrm{e}}+\kappa_{\mathrm{l}}$ is measured and the electronic portion is estimated using the Wiedemann-Franz relation with the balance assumed to be the lattice portion of thermal conductivity. The Wiedemann-Franz law is simply a statement of the relationship between the electronic contribution to thermal transport and the electrical conductivity of a metal and is given by:

$$
\frac{\kappa_{e}}{\sigma}=L T
$$

Where $\mathrm{T}$ is the absolute temperature in Kelvin and $\mathrm{L}$ is the Lorenz constant given by $2.44 \times 10^{-8} \mathrm{~W} \Omega / \mathrm{K}^{2}$. So, given a measurement of electrical conductivity, $\sigma$, and total thermal conductivity, $\kappa$, we can estimate the components of thermal conductivity using Eq. 2 and Eq. 3.

The conversion efficiency is given by the relation

$$
\begin{aligned}
& \text { Efficiency }=\frac{\left(T_{h}-T_{c}\right)(\gamma-1)}{\left(T_{c}+\gamma T_{h}\right)} \\
& \text { where, } \quad \gamma=\sqrt{(1+Z T)}
\end{aligned}
$$

From Eq. 4, it appears that materials with high Figure of Merit (FOM), ZT provides enhanced conversion efficiency. However, ZT is limited by Physics in bulk single and polycrystalline materials and practical devices exceeding $>10 \%$ have not been realized. 


\section{NANOTECHNOLOGY BASED TE MATERIALS}

From Eq. 1, ZT can be increased by Minimizing the thermal conductivity and Maximizing the electrical conductivity. One potential methodology for overcoming limitations of ZT in bulk materials is the development of bulk nanostructures. Nano-structures are engineered material configurations achieved using nanotechnology based techniques. TPG performance (the energy conversion efficiency and power density) can be improved by exploiting the quantum size effects. The power factor, $\mathrm{S}^{2} \sigma$, can be increased due to the quantum effects on electrons and thermal conductivity (k) can be reduced by the interface scattering of phonons. If the figure of merit is improved by a factor of 3 and improved radioisotope based thermal systems are built, then the power generation efficiencies may be increased to 15 to $20 \%$. This potentially will be compatible with the photovoltaic based solar power generation.

In this paper, we are experimenting with bulk nano-structures as a possible path for achieving high conversion efficiencies via increasing the ZT of TE materials.

In producing nano-structures, two important steps are nano-powder production and consolidation of nano-powders. In our case, nano-powders were produced using the Gas Atomization process. The gas atomization process is illustrated in Figure 1 and is described as follows:

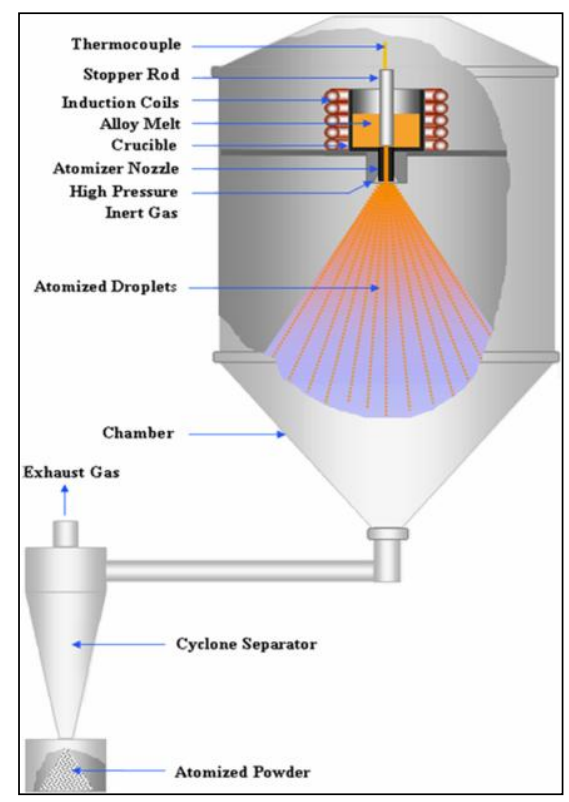

Figure 1. The gas atomization process for nano-powder production.

The alloy of interest gets melted under a vacuum or inert atmosphere in an induction furnace. The melted material is ejected through a nozzle. The ejected material is atomized by a supersonic gas jet. Gas atomization can produce amorphous or nano-structured powders of various materials. Following material processing, shockwave consolidation to fuse nanopowders into a solid while preserving nanostructure is carried out.

For our experiments, a stoichiometric mixture of bismuth, antimony and tellurium (all $99.999 \%$ pure) was gas atomized to produce nanopowders having a make-up of $\mathrm{Bi}_{0.5} \mathrm{Sb}_{1.5} \mathrm{Te}_{3}$, an industry standard chemistry for p-type thermoelectric material ${ }^{3}$. The X-ray diffraction and Electron Back Scatter Diffraction (EBSD) analysis revealed a mix of grain sizes ranging from essentially amorphous to over $150 \mathrm{~nm}$.

\section{SHOCKWAVE CONSOLIDATION OF NANO-POWDERS}

Conventional techniques for nano-powder consolidation, besides using large weights for compacting, sintering techniques have been employed. Among them the widely used one is spark-plasma sintering. Conventional techniques are slow, expensive and of low yield. Using shockwave consolidation by explosives, we have overcome these drawbacks and generate large amounts of consolidated material at low cost and in less time. Furthermore, the motivation for using shockwave consolidation for converting nanopowders into a bulk nano-structured material is to avoid the grain growth that occurs with the heat treatment step of alternative techniques. Figure 2 illustrates the shockwave consolidation technique. 


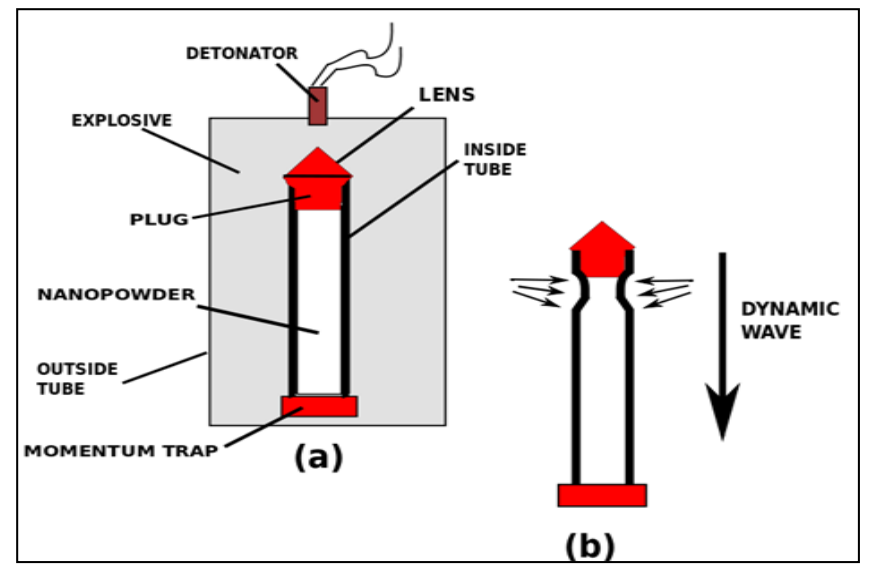

Figure 2. Schematic of consolidation of nano-powders using shockwaves. (a) Cross-section of tube assemblies with nanopowders and explosives. Inside tube has nano-powders, (b) Propagation of shock waves.

Nanopowders, produced using gas atomization of a stoichiometric mixture of the elemental precursors, are inserted into a steel pipe. The p- and n-type nanopowders are separately packed into a steel pipe. This pipe is inserted into a larger pipe. Explosives are added to the interstitial space. The xplosion is set off and consolidated material is ready in a few seconds ${ }^{3}$. Figure 3 shows the outside tube used in our experiments.

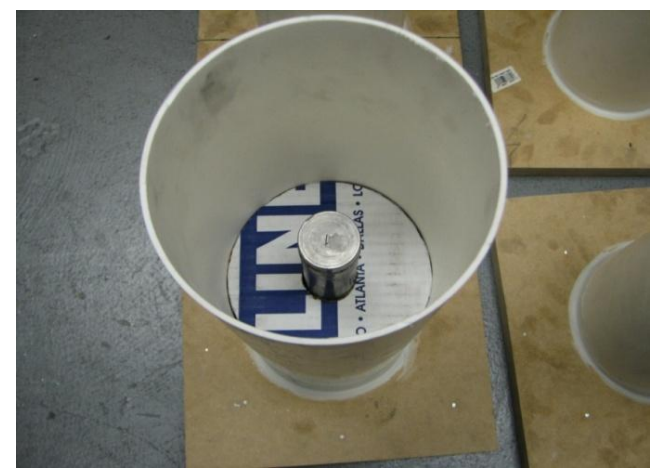

Figure 3. Looking into the outer tube from top. Steel inner tube packed with nano-powders can also be seen.

Under an argon atmosphere, the powder was green compacted into a steel tube which was sealed and placed in an outer cylinder containing ammonium nitrate/fuel oil (ANFO) explosive. Detonation was initiated at the top, causing a pressure pulse in excess of 8 giga Pascal to pass down the tube, consolidating the power into a well-bonded bulk material.

The first shockwave consolidation experiment was carried out in a test site in Socorro, NM and is shown in Figure 4. The explosion shown in Figure 4 had impressive blast characteristics. This experiment of shockwave consolidation of nanopowders resulted into a dense, well-bonded bulk material ${ }^{2}$. Test TPG devices that operate using human body heat differentials were successfully fabricated. Figure 5 shows a typical device with an electronic booster circuit that drives an LED if a thermal gradient of less than $1^{0}$ due to body heat is present. As such, these devices could have applications for operating miniature sensors especially for an astronaut inside a body suit and working inside a constrained environment. Various applications of these devices including road markers have been successfully demonstrated.
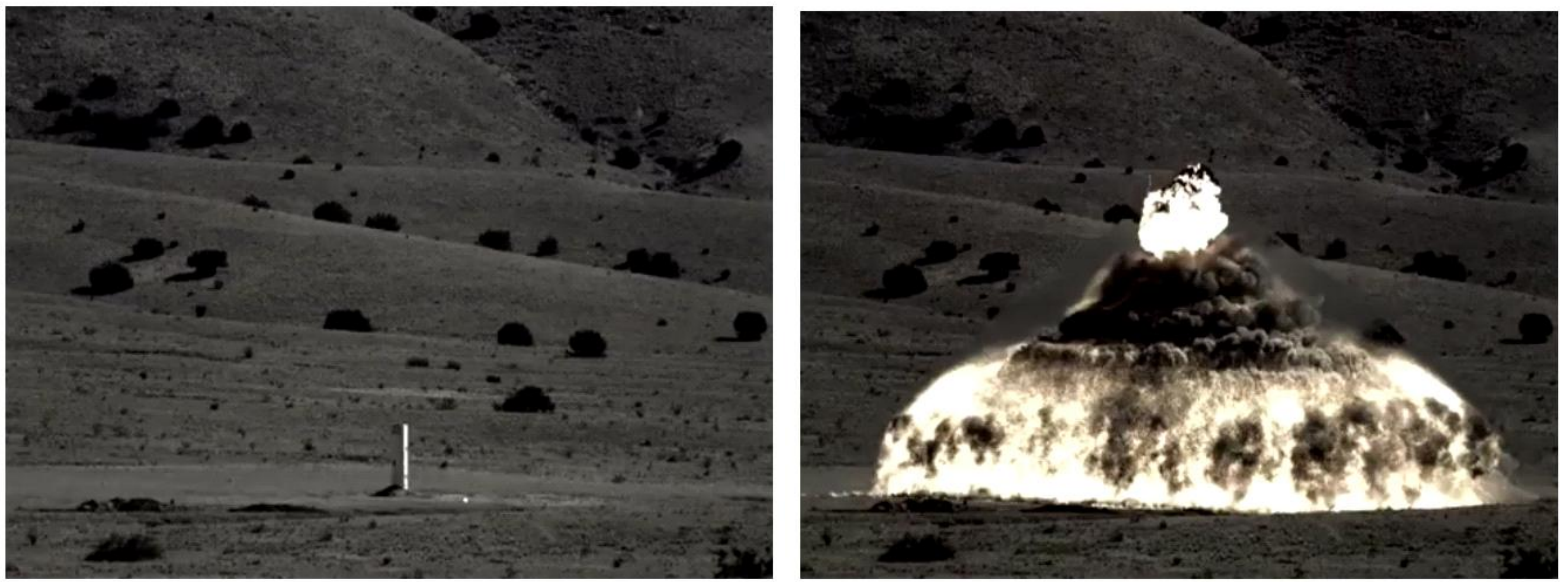

Figure 4. Shockwave consolidation experiment carried out in Socorro, NM. 


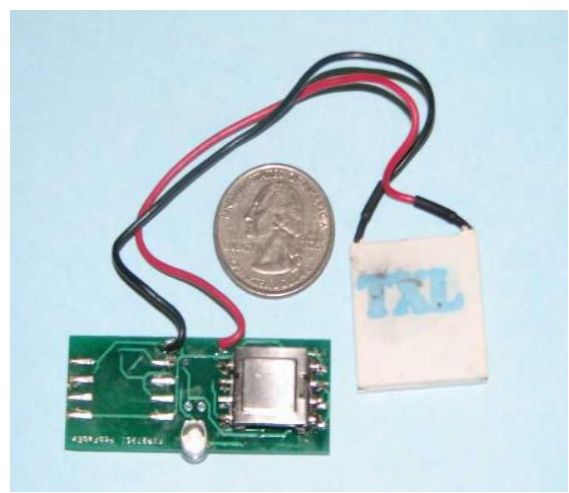

Figure 5. Miniature TPG device that generates electrical current using body heat differential. The passive electronic booster circuit aids in storing and driving an LED.

To further save costs involved in setting up the controlled explosion, the next set of experiments were carried out in a quarry near El Paso, Texas. Figure 6 illustrates the El Paso experiment. For our experiments, bismuth telluride alloy was considered. The p-type thermoelectric material has the chemical formula $\mathrm{Bi}_{0.5} \mathrm{Sb}_{1.5} \mathrm{Te}_{3}$. The n-type thermoelectric material has the formula $\mathrm{Bi}_{2} \mathrm{Te}_{2.7} \mathrm{Se}_{0.3}$. Each of the two materials were prepared as nanopowders using gas atomization and consolidated using the shockwave technique at the El Paso quarry. Samples were cut from the consolidate and sent to the U.S. Army Research Laboratory, Adelphi for independent measurement of TE characteristics.

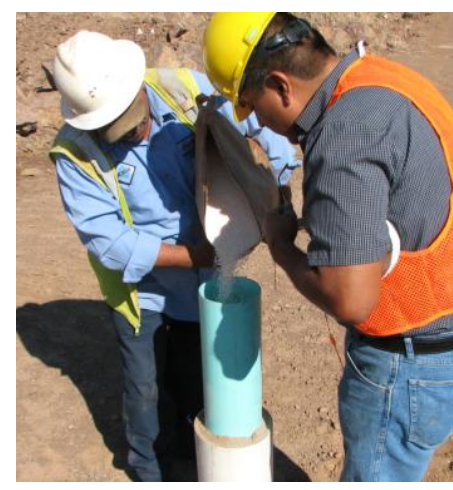

(a)

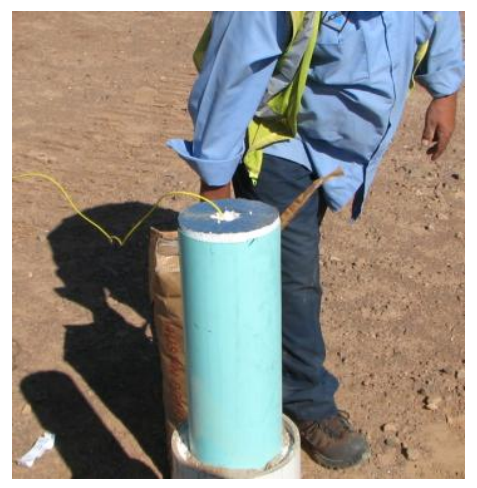

(b)

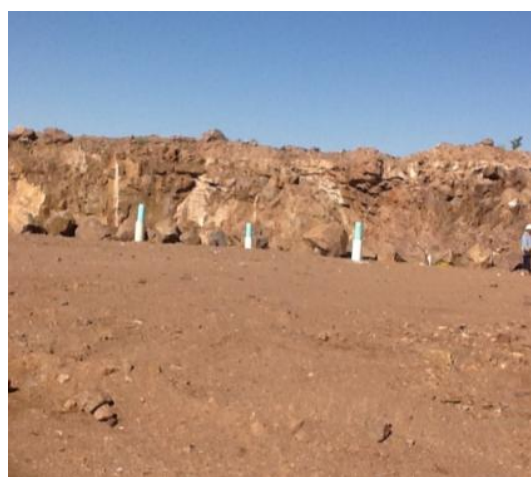

(c)

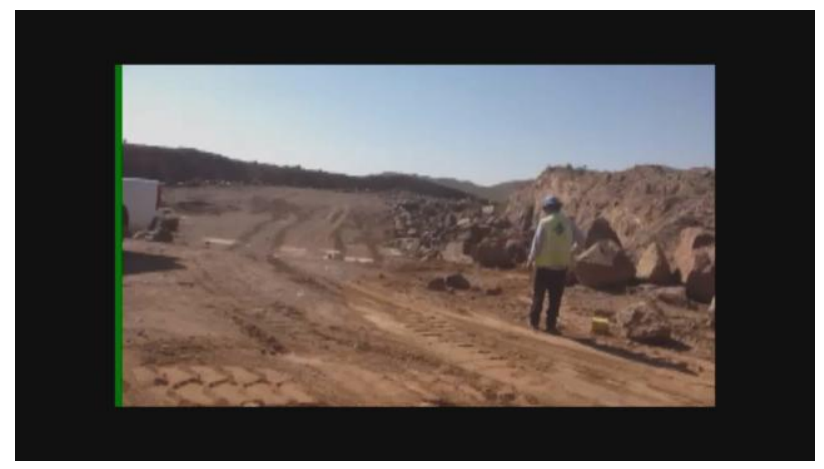

(d)

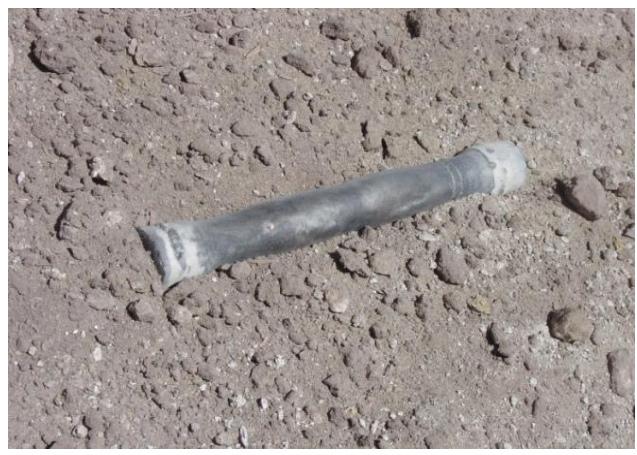

(e)

Figure 6. Shockwave consolidation experiment carried out in an El Paso Quarry. (a) Pouring in explosives inside a PVC tube containing the steel tube with nano-powders. (b) Setting up detonator. (c) Three pipes with explosives and nano-powders ready to be exploded. (d) A scene before initiating the explosion at a safe distance and (e) After explosion, only steel pipe with consolidated nanopowders remains.

\section{SEebeck COEFFicient AND Thermal CONDUCTIVITY}

Figure 7 illustrates the setup used for the measurement of the Seebeck coefficient and thermal conductivity of the newly consolidated material. A piece of compressed steel cylinder with consolidated nano-powder and a slice of the consolidated segment is also shown. This segment was inserted into the sample holder for the measurement of Seebeck coefficient and thermal conductivity. 


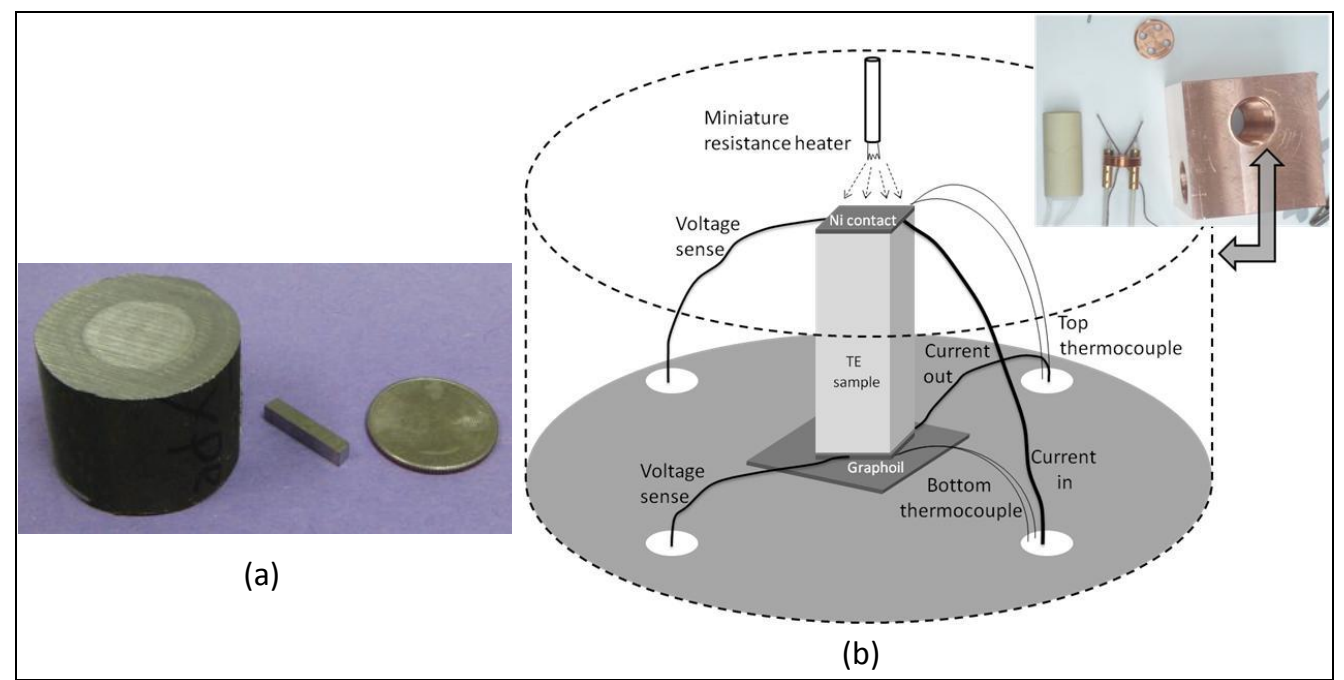

Figure 7. Characterization of consolidated TE nano-powders. (a) Steel pipe segment with consolidated nano-powder in the center. A cut piece of consolidated TE material is also shown. (b) The test set up for measuring thermal conductivity of the sample. The insert shows the sample holder for placing the test sample.

\section{Measurement of Seebeck Coefficient:}

Seebeck is measured by applying a range of temperature differences across the sample, and collecting the corresponding open-circuit voltage data. Figure 8 shows the open circuit voltage measurements as a function of temperature difference.

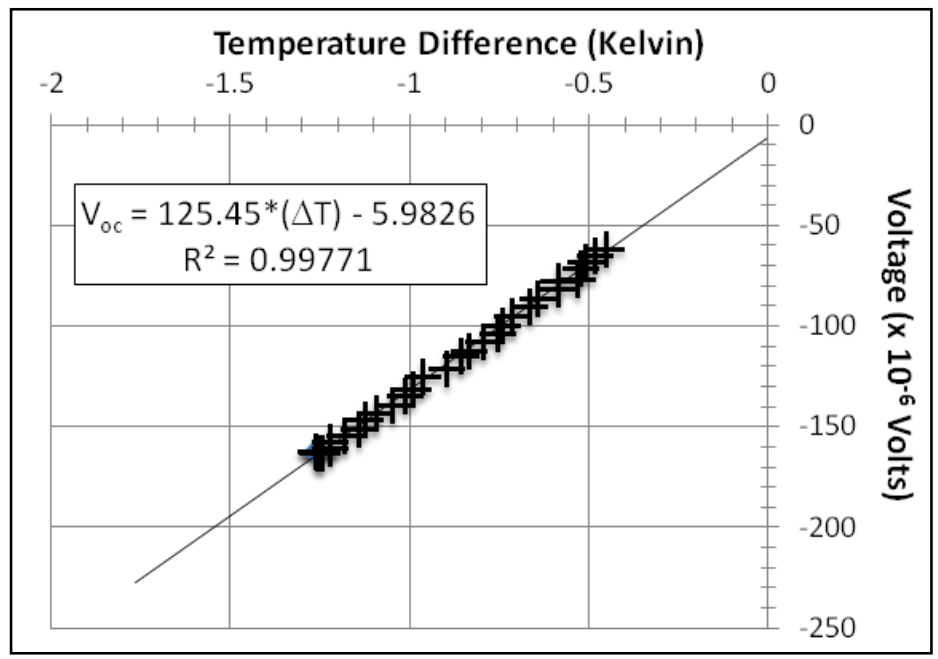

Figure 8. Open circuit voltage vs. temperature differential characteristics for the determination of Seebeck coefficients.

\section{Measurement of Thermal conductivity}

Thermal conductivity determination was done in two parts. In part 1, we applied a steady-state temperature difference across the sample, then passed electrical current through the sample to cause sequential Peltier cooling. Because the current cools the sample, the temperature difference across the sample is reduced as a function of time (See Figure 9). In 
Part 2, replotting that data as a function of corresponding current and measuring the slope yields thermal conductivity, $\mathrm{k}$, as per Eq. 5:

$$
\kappa=\frac{\alpha \mathrm{T}_{\text {contact }}}{\left(\frac{\partial \Delta \mathrm{T}}{\partial \mathrm{I}}\right)\left(\frac{\mathrm{A}}{\ell}\right)}
$$

where $\alpha$ is the Seebeck coefficient, $T_{\text {contact }}$ is the temperature of the contact, $A / \ell$ is the area-to-length ratio of the sample and the $\partial \Delta \mathrm{T} / \partial \mathrm{I}$ is the slope of the data. The temperature differential as a function of current characteristics is shown in Figure 10 .

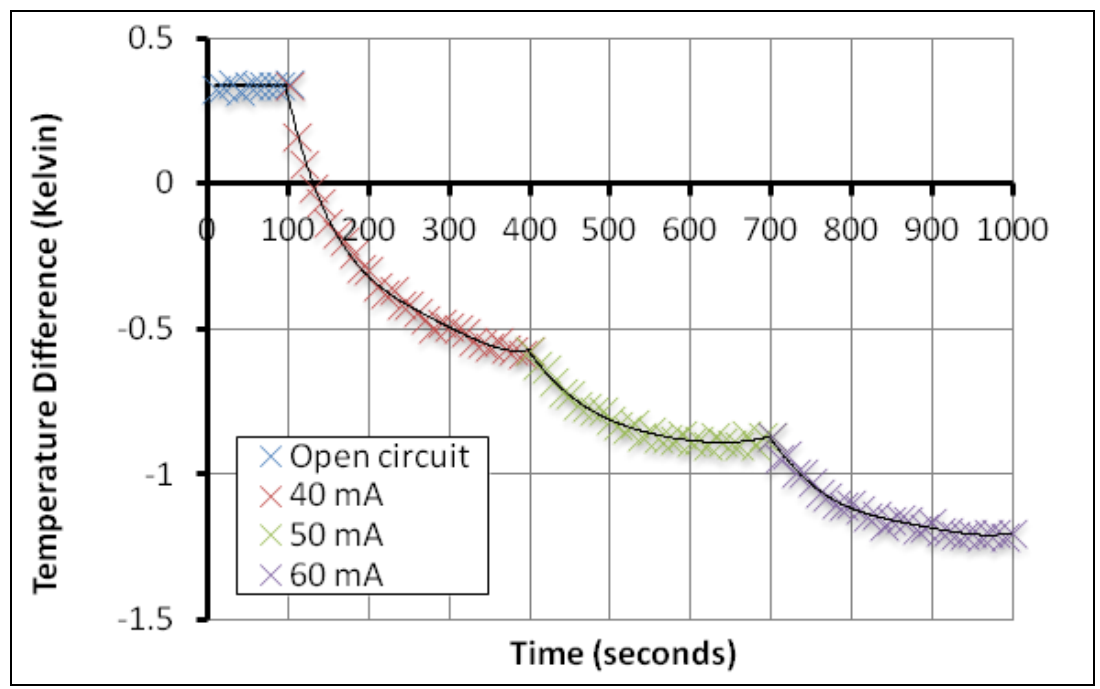

Figure 9. Data as a function of current.

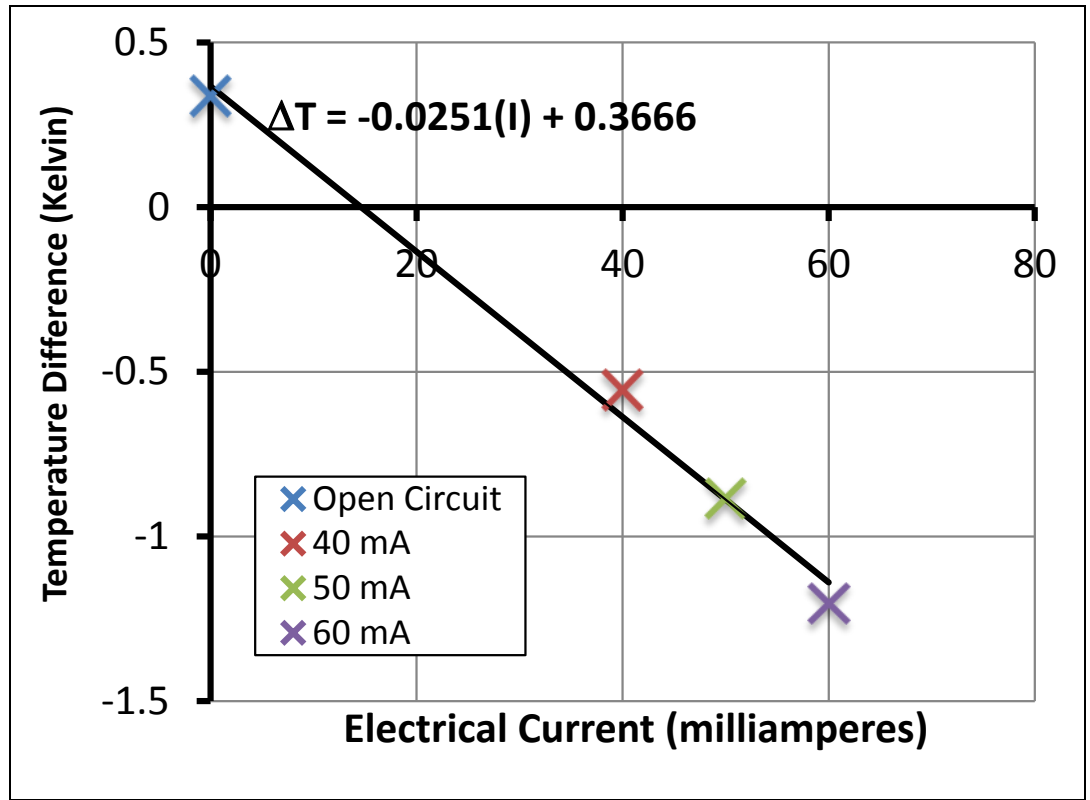

Figure 10. Temperature differential as a function of current 
Table 1 and 2 summarizes the parameters derived from measurements with melt grown crystalline materials for $\mathrm{n}$ and $\mathrm{p}$ type materials. The consolidated samples showed a decrease in thermal conductivity for the p-type material, even while resulting in an increase in electrical conductivity. This suggests that the lattice component, $\kappa_{1}$, is undergoing a significant reduction. We believe that we are seeing some unexpected physics associated with the electrical conductivity and that the Wiedemann-Franz relation may not accurately characterize electronic thermal transport for this material. For both the p-type and the n-type samples, a factor of two increase in electrical conductivity was obtained as contrasted to the crystalline sample. This is a very surprising result.

Table 1: Comparison between shockwave and melt grown crystalline of the p-type $\mathrm{Bi}_{0.5} \mathrm{Sb}_{1.5} \mathrm{Te}_{3}$ thermoelectric material.

\begin{tabular}{|l|l|l|l|l|l|l|}
\hline Material & $\begin{array}{l}\mathrm{S} \\
(\mu \mathrm{V} / \mathrm{K})\end{array}$ & $\begin{array}{l}\sigma \\
(\mathrm{S} / \mathrm{m})\end{array}$ & $\begin{array}{l}\mathrm{K} \\
(\mathrm{W} / \mathrm{mK})\end{array}$ & $\begin{array}{l}\mathrm{K}_{\mathrm{e}} \\
(\mathrm{W} / \mathrm{mK})\end{array}$ & $\begin{array}{l}\mathrm{K}_{\mathrm{ph}} \\
(\mathrm{W} / \mathrm{mK})\end{array}$ & $\begin{array}{l}\mathrm{ZT} \\
294 \mathrm{~K}\end{array}$ \\
\hline $\begin{array}{l}\text { Shock Cons. Nanopowder } \\
(\text { ARL measurements) }\end{array}$ & +125 & 173,000 & 1.21 & 1.24 & $\mathrm{~N} / \mathrm{A}$ & 0.66 \\
\hline Melt Grown Crystalline & +208 & 90,000 & 1.4 & 0.65 & 0.75 & 0.82 \\
\hline
\end{tabular}

Table 2: Comparison between shockwave consolidated and melt grown crystalline material for the $n$-type $\mathrm{Bi}_{2} \mathrm{Te}_{2.7} \mathrm{Se}_{0.3}$ thermoelectric Material

\begin{tabular}{|l|l|l|l|l|l|l|}
\hline Material & $\begin{array}{l}\mathrm{S} \\
(\mu \mathrm{V} / \mathrm{K})\end{array}$ & $\begin{array}{l}\sigma \\
(\mathrm{S} / \mathrm{m})\end{array}$ & $\begin{array}{l}\mathrm{K} \\
(\mathrm{W} / \mathrm{mK})\end{array}$ & $\begin{array}{l}\mathrm{K}_{\mathrm{e}} \\
(\mathrm{W} / \mathrm{mK} \\
)\end{array}$ & $\begin{array}{l}\mathrm{K}_{\mathrm{ph}} \\
(\mathrm{W} / \mathrm{mK})\end{array}$ & $\begin{array}{l}\mathrm{ZT} \\
294 \mathrm{~K}\end{array}$ \\
\hline $\begin{array}{l}\text { Shock Cons. Nanopowder } \\
\text { (ARL measurements) }\end{array}$ & -131 & 151,800 & 1.93 & 1.1 & 0.83 & 0.40 \\
\hline Melt Grown Crystalline & -222 & 90,000 & 1.5 & 0.65 & 0.85 & 0.87 \\
\hline
\end{tabular}

\section{SUMMARY AND CONCLUSIONS}

In this paper, consolidation of nano-powders using the shockwave technique with a controlled explosion is explored. The shockwave technique offers a path to obtain consolidated nano-structured material quickly in large quantities and at low cost. We distinguish our technique, explosive shockwave consolidation, from the spark plasma sintering approach that it retains the small feature sizes that are desirable. We have successfully demonstrated a reduction in thermal conductivity and found a surprising result in the substantial increase in electrical conductivity. This presents a road map for the path ahead. A higher ZT material is the goal of our research. The industry standard stoichiometries are selected to be heavily doped in order to have reasonably good electrical conductivity. If we can reduce the doping, it should allow higher thermopowers, with a reduction in electrical conductivity, which we can now tolerate. These results are being further analyzed

\section{REFERENCES}

1. “Thermoelectrics Handbook: Macro-Nano,” Ed: D.M. Rowe, CRC Taylor \& Francis, Boca Raton, FL, 2006.

2. B. Poudel, . Hao, Y. Ma, Y. Lan, A. Minnich, B. Yu, X. Yan, D. Wang, A. Muto, D. Vashaee, X. Chen, J. Liu, M. Dresselhaus, G. Chen and Z. Ren, "High-Thermoelectric Performance of Nanostructured Bismuth Antimony Telluride Bulk Alloys", Science, Vol 320, (2008): 634-641.

3. Jan Beck, Manuel Alvarado, David Nemir, Mathew Nowell, Lawrence Murr, and Narasimha Prasad., "ShockWave Consolidation of Nanostructured Bismuth Telluride Powders," Journal of Electronic Materials, Volume 41, Issue 6, pp 1595-1600, June 2012. 